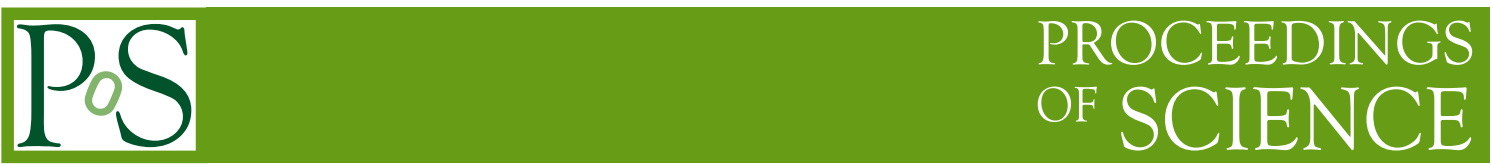

\title{
Inclusive jet results from the CMS experiment
}

\author{
Suman Chatterjee* \\ on behalf of the CMS collaboration, \\ Department of High Energy Physics, Tata Institute of Fundamental Research, \\ 1 Homi Bhabha Road, Mumbai, 400005, India \\ E-mail: suman. chatterjeedcern.ch, suman.chatterjeedtifr.res.in
}

\begin{abstract}
The CMS experiment has measured the cross section of inclusive jets in proton-proton collisions with center-of-mass (COM) energies of 7, 8, and $13 \mathrm{TeV}$ after the precise calibration of jet momentum. At $7 \mathrm{TeV}$ COM energy, the ratio of the double-differential cross section of inclusive anti- $k_{\mathrm{T}}$ jets with distance parameter $R$ of 0.5 to that of anti- $k_{\mathrm{T}}$ jets with $R$ of 0.7 was measured as a function of transverse momentum $p_{\mathrm{T}}$ and rapidity $y$ of the jet. This was followed by the measurement of the ratio of the inclusive anti- $k_{\mathrm{T}}$ jet cross sections as a function of jet $p_{\mathrm{T}}$ and $y$, for values of $R$ ranging from 0.1 to 1.2 to that using a $R$ of 0.4 , and the study of the dependence of the inclusive jet production cross section on the parameter $R$ using $35.9 \mathrm{fb}^{-1}$ data collected at $13 \mathrm{TeV}$ COM energy. The results are compared to calculations at leading order (LO) and next-toleading order (NLO) in the strong coupling constant using different parton shower models. The variation of the ratio of cross sections with the distance parameter is described well by calculations including a parton shower model, but not by a pure leading order QCD calculation including nonperturbative effects. The descriptions of the ratios of cross sections are significantly improved when NLO QCD calculations with nonperturbative effects are used.
\end{abstract}

European Physical Society Conference on High Energy Physics - EPS-HEP2019 -

10-17 July, 2019

Ghent, Belgium

${ }^{*}$ Speaker. 


\section{Introduction}

Quantum Chromodynamics (QCD) is a gauge theory describing the strong interaction between quarks and gluons. A jet is an algorithmic reconstruction using energy clusters from hadrons produced in the fragmentation of partons created in short-distance scattering. The production cross sections for high transverse momentum $\left(p_{\mathrm{T}}\right)$ partons can be calculated using perturbative QCD (pQCD). Predictions for hadron production need, in addition, models for parton showering and nonperturbative (NP) effects, such as hadronization, underlying events (UE), which consist of the interaction of multiple partons from the two colliding protons, and color connection to beam remnants.

In the CMS experiment [1], the particle-flow algorithm [2] aims to reconstruct and identify each individual particle in an event, with an optimized combination of information from the various elements of the CMS detector. For each event, hadronic jets are clustered from these reconstructed particles using the infrared- and collinear-safe anti- $k_{\mathrm{T}}$ algorithm [3], as implemented in the FASTJET package [4]. The jet momentum is determined as the vectorial sum of all particle momenta in the jet, and is found from simulation to be, on average, within 5 to $10 \%$ of the momentum of the particle-level jets, reconstructed using stable particles (lifetime $>30 \mathrm{ps}$ ) excluding neutrinos, for jet $p_{\mathrm{T}}>50 \mathrm{GeV}$ and rapidity $|y|<2.5$. Tracks, within the jet, identified to be originating from pileup vertices are discarded and an offset correction is applied to correct for remaining contributions from additional proton-proton collisions in the same bunch crossing [5]. Additional selection criteria are used to remove jets potentially dominated by instrumental effects or reconstruction failures.

In this note, we discuss the measurement by the CMS experiment of the absolute cross section of inclusive jets in proton-proton collisions at center-of-mass (COM) energy of $13 \mathrm{TeV}$ [6], the measurement of the ratio of cross sections of inclusive jets of different sizes at COM energies of 7 [7], and $13 \mathrm{TeV}$ [8]. The former measurement is useful to determine the parton distribution function (PDF) of gluons carrying a large share of the proton momentum, to measure the coupling constant of strong interaction $\left(\alpha_{S}\right)$, and also to understand $\mathrm{pQCD}$, while the latter ones are sensitive to the details of the theoretical modeling of the perturbative and NP processes involved in the evolution of the partons.

\section{Inclusive jet cross section at $13 \mathrm{TeV}$}

The cross section has been measured for inclusive anti- $k_{\mathrm{T}}$ jets using distance parameters of 0.4 (AK4), and of 0.7 (AK7) at particle level, after unfolding the measurements made at detector level, and compared to the fixed order prediction at NLO, computed using NLOJet++ [9], and to predictions from Monte Carlo (MC) simulations. For the fixed order prediction, as it is at the parton level, a NP correction is applied; this correction is derived using MC samples with LO PYTHIA [10] and HERWIG++ [11] generators and the corresponding parton showers, and also with NLO generator POWHEG [12] accompanied by PYTHIA parton shower.

As shown in Fig. 1, fixed order calculations at NLO can describe the AK7 jet cross section well within systematic uncertainties, but overpredict the cross section of AK4 jets. Also note that MMHT and NNPDF3.0 PDF sets give very similar predictions as the ones by CT14, whereas HERAPDF gives a smaller prediction specially at high jet $p_{\mathrm{T}}$ owing to a smaller gluon fraction at large 

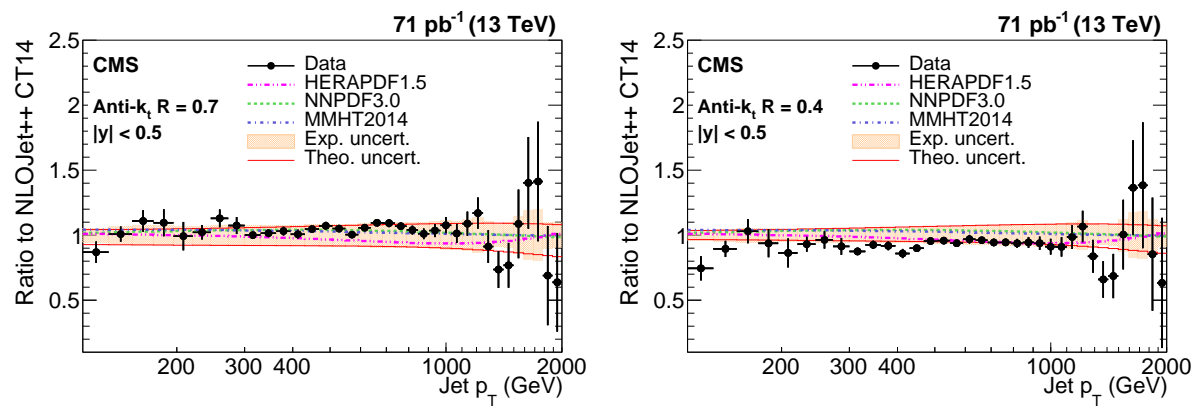

Figure 1: Ratio of measured values of cross section for AK7 (left) and AK4 (right) jets to predictions from fixed order calculation using different PDF sets. The error bars correspond to the statistical uncertainties of the data and the shaded bands to the total experimental systematic uncertainties [6].
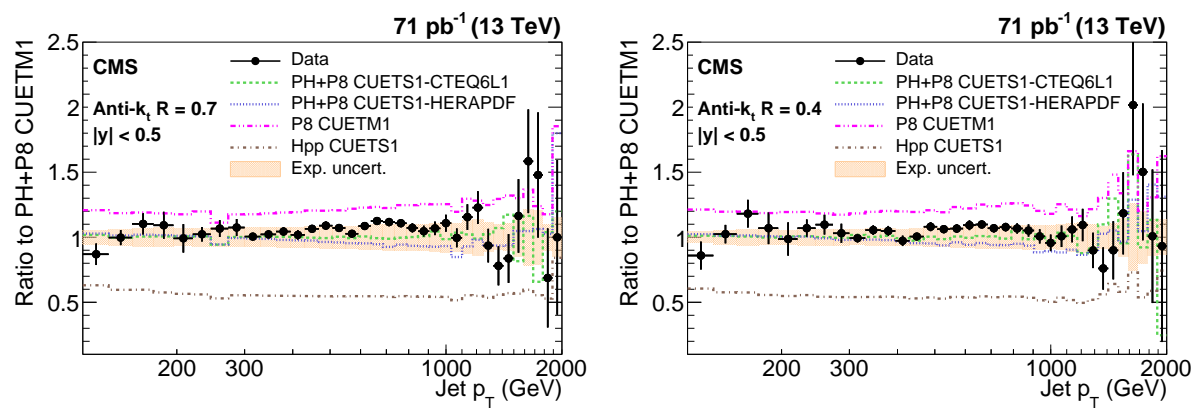

Figure 2: Ratio of measured values of cross section for AK7 (left) and AK4 (right) jets to predictions from different MC generators. The error bars correspond to the statistical uncertainties of the data and the shaded bands to the total experimental systematic uncertainties [6].

Bjorken scale. In Fig. 2, one can notice that, both for AK4 and AK7 jets, PYTHIA overpredicts, and HERWIG++ underpredicts the normalization of the production cross section as compared to that in data, although HERWIG++ can describe the shape of the variation of cross section as a function of $p_{\mathrm{T}}$ of jets. PYTHIA also does the same, but only up to jet $p_{\mathrm{T}}$ of moderate values. POWHEG, accompanied by PYTHIA parton shower, can describe both the shape and normalization of the differential cross sections of AK4 and AK7 jets as seen in the data well within systematic uncertainties.

\section{Ratio of inclusive jet cross sections at $7 \mathrm{TeV}$}

The ratio of the double-differential cross section of AK5 jets has been taken with respect to that of AK7 jets at particle level at $7 \mathrm{TeV}$ of COM energy. As some of systematic uncertainties cancel in the ratio, it is more sensitive to perturbative and NP effects; thus theory predictions are put to a more stringent test to data than the absolute cross section measurement. Fig. 3 shows that the NLO prediction for the ratio, along with nonperturbative corrections can describe the trend of the variation of the ratio as a function of jet $p_{\mathrm{T}}$, but predicts a slightly larger normalization. The POWHEG generator, accompanied by PYTHIA parton shower, can describe the ratio very well. PYTHIA describes the ratio in data well in the low $p_{\mathrm{T}}$ region, whereas HERWIG++ describes the same well in high $p_{\mathrm{T}}$ values of jets. 


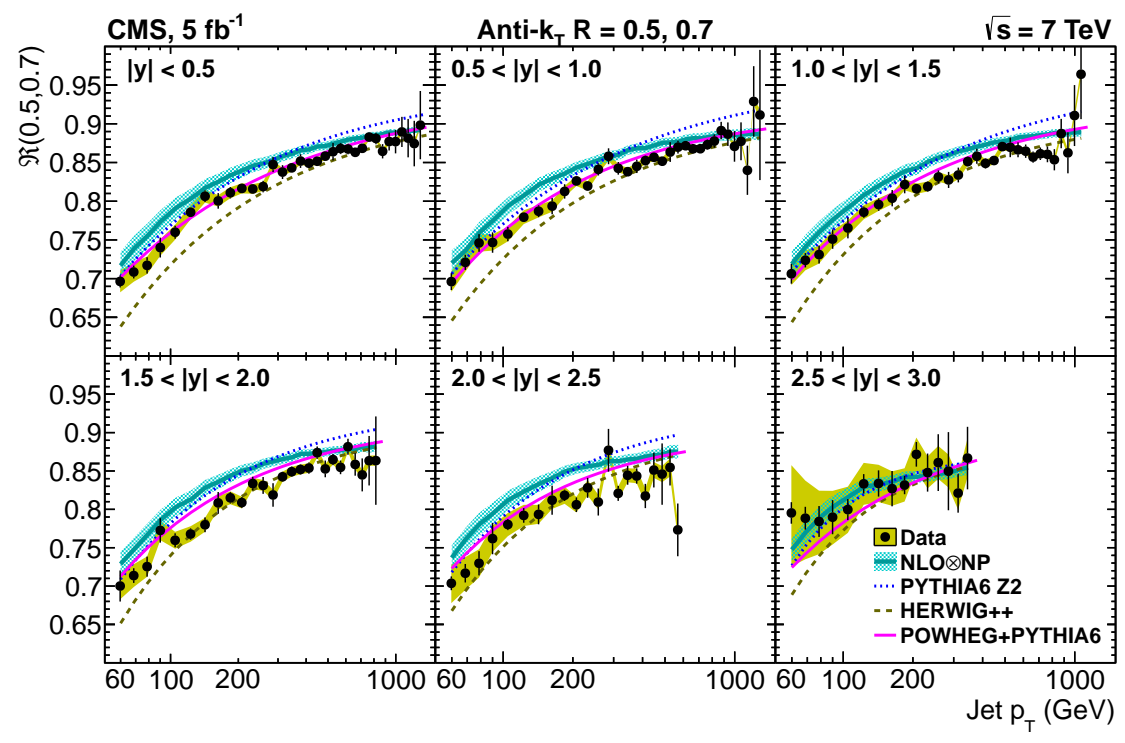

Figure 3: Ratio of cross section of AK5 and AK7 jets in six rapidity bins up to $|y|=3.0$, compared to predictions from fixed order calculation at NLO and MC generators. The error bars on the data points represent the statistical and uncorrelated systematic uncertainty added in quadrature, and the shaded bands represent the correlated systematic uncertainty [7]. The NLO calculation was provided by G. Soyez [13].

\section{Ratios of inclusive jet cross sections at $13 \mathrm{TeV}$}

Anti- $k_{\mathrm{T}}$ jets are reconstructed using different values of $R$, from 0.1 to 1.2. The ratio of cross sections are measured for all these jets with respect to that of AK4 jets as a function of jet $p_{\mathrm{T}}$ for different ranges of the rapidity of the jet. At low values of $R$, more perturbative radiation, and also particles from hadronization escape the catchment area of jets, for large $R$ values, UE contaminate the jet more with soft radiation; thus the measurement of jets of different $R$ values can provide insight on both perturbative and NP aspects of QCD. In CMS, standard jet energy corrections are derived only for AK4 and AK8. The calibration factors for AK4 jets are applied on AK1 to AK6 jets, for larger jet sizes calibration factors for AK8 jets are used; in order to account for the difference in the distance parameter, an extra correction factor is determined using simulated inclusive jet samples. The experimental uncertainty consists of systematic uncertainties from jet energy scale, jet energy resolution, pileup, and also from additional correction just mentioned. Apart from the systematic uncertainties due to experimental sources, theory calculations and generators have uncertainties in their predictions for the cross section ratio. For the fixed-order predictions, the contributing factors include the choice of renormalization and factorization scales, from the PDF uncertainty, the uncertainty from $\alpha_{S}$, and the uncertainty due to the NP corrections. The statistical uncertainty for the cross section ratio has been evaluated using the Jackkniffe method using ten sub-samples with exactly the same amount of data. The experimental systematic uncertainty for the cross section ratio is of similar size as the statistical uncertainty, and the theory uncertainty is dominated by the choice of the renormalization and factorization scales.

Fig. 4 shows that the prediction from the POWHEG generator, accompanied by PYTHIA parton shower, can describe the ratio reasonably well, except at low jet $p_{\mathrm{T}}$ for large values of $R$, for which 


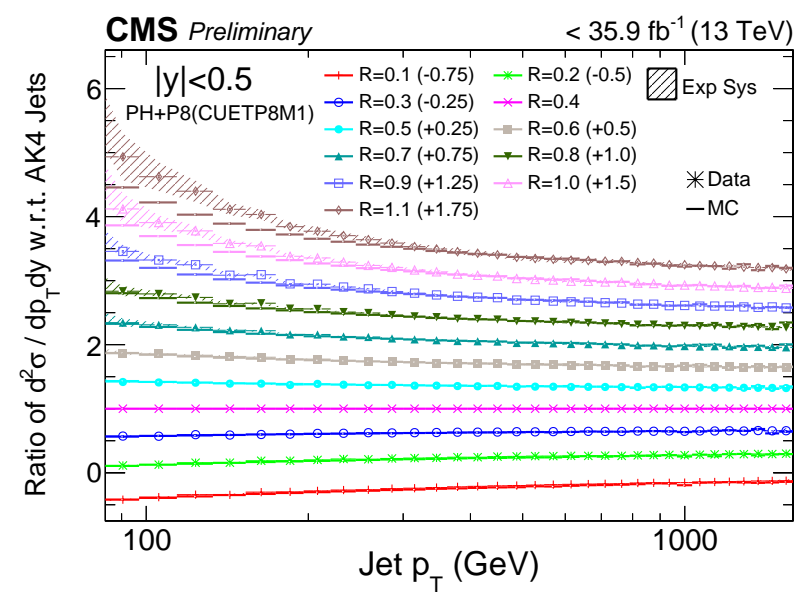

Figure 4: Comparison of the ratio of differential cross section of jets of different sizes with respect to that of AK4 jets from data and from NLO predictions using POWHEG + PYTHIA (CUETP8M1 tune [14]) in the region $|y|<0.5$. Colored symbols indicate data and colored lines represent prediction from simulation. Offsets by amount written in the parentheses have been added to the corresponding data points to separate the results for different jet sizes [8].

experimental uncertainties are also larger than those for lower values of $R$. In Fig. 5, comparisons
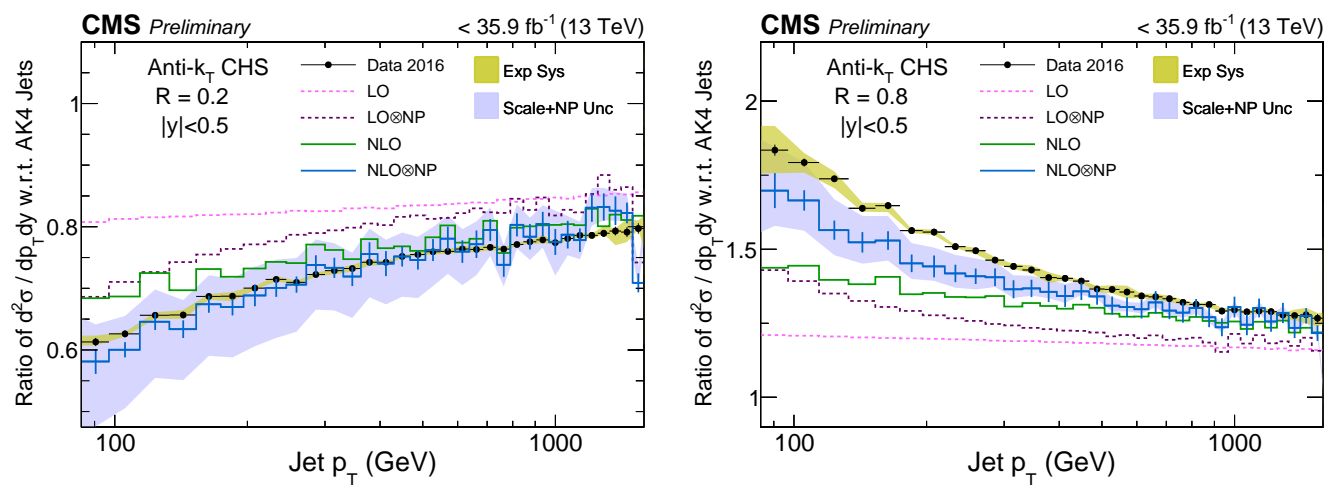

Figure 5: Comparison of the ratio of differential cross section of the AK2 (left) and AK8 (right) jets with respect to that of AK4 jets from data and PQCD predictions using NLOJET++ in the region $|y|<0.5$. Black symbols indicate data and colored lines represent pQCD predictions. Statistical uncertainties are shown as vertical bars for the data and the NLO prediction with nonperturbative correction; yellowish olive region around data represents experimental systematic uncertainty whereas region shaded in light blue color around $\mathrm{NLO} \otimes \mathrm{NP}$ prediction shows the theory uncertainty in the prediction [8].

are made for the ratio of cross section for $\mathrm{AK} 2$ and $\mathrm{AK} 8$ jets with respect to that of $\mathrm{AK} 4$ jets respectively with fixed order PQCD predictions at LO and NLO. NP corrections are derived from MC samples with the POWHEG generator, and PYTHIA and HERWIG++ parton showers respectively. For AK2 jets, after computing the ratio at NLO and taking into account NP corrections, the theory prediction can describe the data well, where as for AK8 jets theory prediction underpredicts the ratio, as observed in data, till high values of jet $p_{\mathrm{T}}$. In both the cases, the description is significantly improved from LO to NLO. Fig. 6 shows the ratio of cross sections of inclusive jets with respect 

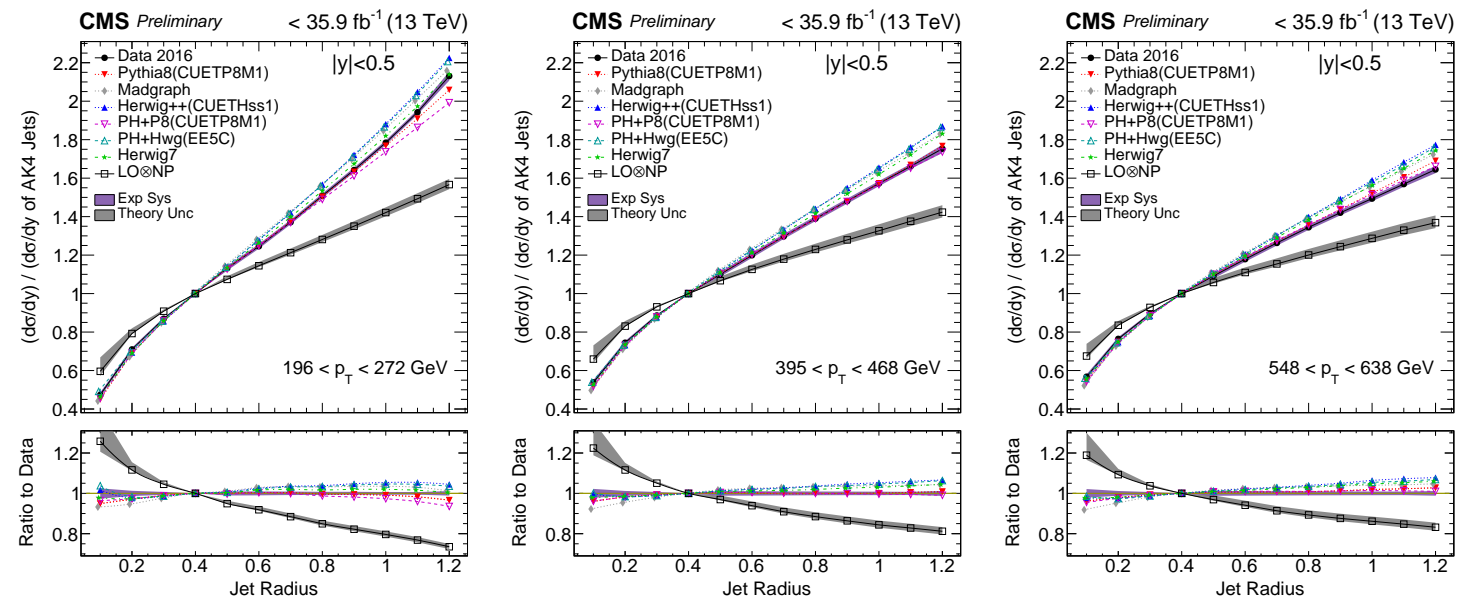

Figure 6: Comparison of the ratio of cross sections of inclusive jets of various sizes with respect to AK4 jets, as a function of jet size in different regions of jet $p_{\mathrm{T}}$ in data and from several theoretical predictions in rapidity bins $|y|<0.5$ at particle level. When the cross section ratio is taken between fixed NLO predictions for two jet sizes, the ratio becomes $\mathrm{LO}$ at $\alpha_{S}$, this is quoted as $\mathrm{LO} \otimes \mathrm{NP}$ in the figure. Experimental uncertainties on the ratio of cross sections are shown with bands around the data points, while theory uncertainties are shown with the bands around the fixed-order predictions [8].

to that of AK4 jets in different ranges of jet $p_{\mathrm{T}}$ as a function of $R$. The key observation here is that the fixed order prediction, where the cross section is computed at NLO, and hence the ratio is only at $\mathrm{LO}$, predicts a different trend for the variation of the cross section ratio with $R$ as compared to the trend observed in data, and also in predictions from MC simulations.

\section{Conclusion}

This note discusses a measurement of the double-differential cross section of anti- $k_{\mathrm{T}}$ jets as a function of jet $p_{\mathrm{T}}$ and absolute rapidity $|y|$ for distance parameters $R=0.4$ (AK4) and 0.7 (AK7) using data from proton-proton collisions at $\sqrt{s}=13 \mathrm{TeV}$ collected with the CMS detector. Leading order Monte Carlo generators show significant discrepancies, mainly in normalization, in the description of absolute cross section of jet production, which is well described by next-to-leading order (NLO) generators. Fixed order prediction at NLO describes the absolute cross section of AK7 jets well, but overestimates the same for AK4 jets. Measurement of the ratio of cross sections of inclusive AK5 and AK7 jets is best described by matching the cross section prediction at NLO with parton showers. This letter also mentions the first measurement of the ratio of cross sections of inclusive anti- $k_{\mathrm{T}}$ jets of multiple sizes w.r.t. AK4 jets by the CMS experiment. From the ratio measurement, it is observed that the NP correction is important to describe the data at low transverse momentum. Thus, the modelling of nonperturbative effects, such as hadronization and the underlying event, and also of perturbative radiation have significant impact on the description of the data in different regions of phase space. The variation of the ratio of cross sections with $R$ emphasizes the importance of parton shower to capture the effects of higher order terms in the perturbation series by the resummation approach, which is absent in the case of fixed-order computation. Therefore, this study shows the importance of final-state radiation modelled in Monte Carlo 
simulation to describe the data, and also points that the differences between various parton shower and hadronization models are significant.

\section{References}

[1] CMS Collaboration, S. Chatrchyan et al., The CMS experiment at the CERN LHC, JINST 3 (2008) S08004.

[2] CMS Collaboration, A. M. Sirunyan et al., Particle-flow reconstruction and global event description with the CMS detector, JINST 12 (2017), no. 10 P10003, [arXiv: 1706.04965 ].

[3] M. Cacciari, G. P. Salam, and G. Soyez, The Anti-k(t) jet clustering algorithm, JHEP 04 (2008) 063, [arXiv:0802.1189].

[4] M. Cacciari, G. P. Salam, and G. Soyez, FastJet User Manual, Eur. Phys. J. C72 (2012) 1896, [arXiv:1111.6097].

[5] CMS Collaboration, V. Khachatryan et al., Jet energy scale and resolution in the CMS experiment in pp collisions at 8 TeV, JINST 12 (2017), no. 02 P02014, [arXiv: 1607.03663 ].

[6] CMS Collaboration, V. Khachatryan et al., Measurement of the double-differential inclusive jet cross section in proton-proton collisions at $\sqrt{s}=13 \mathrm{TeV}$, Eur. Phys. J. C76 (2016), no. 8451 , [arXiv:1605.04436].

[7] CMS Collaboration, S. Chatrchyan et al., Measurement of the ratio of inclusive jet cross sections using the anti- $k_{T}$ algorithm with radius parameters $R=0.5$ and 0.7 in pp collisions at $\sqrt{s}=7 \mathrm{TeV}$, Phys. Rev. D90 (2014), no. 7 072006, [arXiv: 1406.0324 ].

[8] CMS Collaboration, Measurement of the dependence of inclusive jet production cross sections on the anti- $k_{\mathrm{T}}$ distance parameter in proton-proton collisions at sqrt(s) $13 \mathrm{TeV}$, Tech. Rep.

CMS-PAS-SMP-19-003, CERN, 2019, [http://cds . cern. ch/record/2126325].

[9] Z. Nagy, Next-to-leading order calculation of three jet observables in hadron hadron collision, Phys. Rev. D68 (2003) 094002, [hep-ph/ 0307268$].$

[10] T. Sjöstrand, S. Ask, J. R. Christiansen, R. Corke, N. Desai, P. Ilten, S. Mrenna, S. Prestel, C. O. Rasmussen, and P. Z. Skands, An Introduction to PYTHIA 8.2, Comput. Phys. Commun. 191 (2015) 159-177, [arXiv:1410.3012].

[11] M. Bahr et al., Herwig++ Physics and Manual, Eur. Phys. J. C58 (2008) 639-707, [arXiv:0803.0883].

[12] S. Frixione, P. Nason, and C. Oleari, Matching NLO QCD computations with Parton Shower simulations: the POWHEG method, JHEP 11 (2007) 070, [arXiv: 0709. 2092].

[13] G. Soyez, A Simple description of jet cross-section ratios, Phys. Lett. B698 (2011) 59-62, [arXiv:1101.2665].

[14] CMS Collaboration, V. Khachatryan et al., Event generator tunes obtained from underlying event and multiparton scattering measurements, Eur. Phys. J. C76 (2016), no. 3 155, [arXiv:1512.00815]. 